\title{
Edvard Munch: enfermedad y genialidad en el gran artista noruego
}

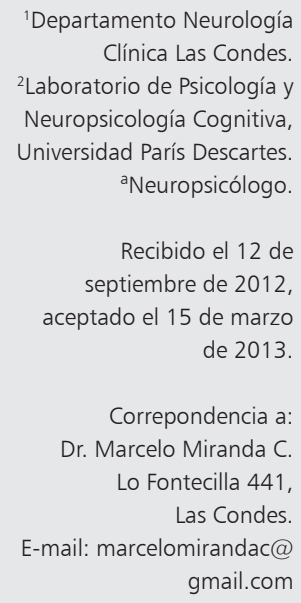

\author{
MARCELO MIRANDA C. ${ }^{1}$, EVA MIRANDA C., MATÍAS MOLINA D. ${ }^{2, a}$
}

\begin{abstract}
Edvard Munch is one the most transcendental artists of all times. His work is innovative in terms of reflecting the grief, sadness, loneliness and the impact of death in human beings as no one did it before. Behind his work it is possible to find many clues given by Munch himself of the reason of his creativity: a childhood surrounded by death and sorrow, and the development of an affective disorder that led him to alcoholism and many hospitalizations due to psychotic episodes. In this review, we analyze Munch's life and his disease that undoubtedly contributed to his great artistic legacy.

(Rev Med Chile 2013; 141: 774-779).
\end{abstract}

Key words: Affective disorders, psychotic; Edvard Munch, creativity.
"Estaba al borde de la locura: era sólo tocar y caer". "Así como Leonardo estudió la anatomía humana y disecó cuerpos, yo trato de disecar almas". "Mis problemas son parte de mí y por lo tanto de mi arte. Ellos son indistinguibles de mí, y su tratamiento destruiría mi arte. Quiero mantener esos sufrimientos".

\section{Edvard Munch}

$\mathrm{E}$ dvard Munch (Loten, Noruega. 1863-1944, Ekely, Noruega), fue uno de los pintores fundadores del movimiento expresionista. Constituye, junto a Henrik Ibsen y Eduard Grieg, el trío de los más grandes artistas noruegos. Sus padres fueron el médico militar Christian Munch y Laura Christine. Las tempranas pérdidas de su madre y su hermana Sophie debido a una tuberculosis, marcaron su infancia y adolescencia e influyeron para siempre su vida y su expresión artística en la que se describe la experiencia, el dolor, la muerte, la soledad, la angustia y la inevitable vejez $^{1-6}$. Cuadros como "La niña enferma" (18851886) (Figura 3), "Muerte en la pieza del enfermo" (1895), "La madre muerta y la Niña” (1897-1899), con obsesivas repeticiones y variaciones demuestran el profundo e insoslayable impacto que estos sucesos tuvieron en su ser.
A los 21 años, se acerca a los círculos bohemios de Oslo, con ideas avanzadas en cuanto a liberalidad sexual y artística. Inició estudios de ingeniería para abandonarlos por la pintura y luego hacer múltiples viajes a París para conocer la obra de Van Gogh, Toulouse Lautrec, Gauguin y Whistler, entre otros. Su obra evolucionó del naturalismo, luego al simbolismo y finalmente a un estilo propio: el expresionismo ${ }^{6}$. En sus primeras obras es notoria la influencia de estos artistas especialmente Gauguin, por la simpleza de las formas y el uso de colores poco convencionales.

La escasa literatura que existe sobre los factores biográficos y médicos que incidieron en la obra de Munch ha sido la motivación para escribir este artículo que pretende ser un pequeño aporte y a la vez un modesto homenaje a este gran artista.

\section{Enfermedad en Munch}

Son las propias notas de Munch las que nos muestran el significado de su arte. En una edad avanzada decía: "sin temor ni enfermedad, mi vida habría sido como un barco sin timón" $"$ ",6.

Podemos hacer un paralelismo entre Munch y Van Gogh: ambas vidas marcadas por el dolor y la angustia y muy probablemente por una enferme- 


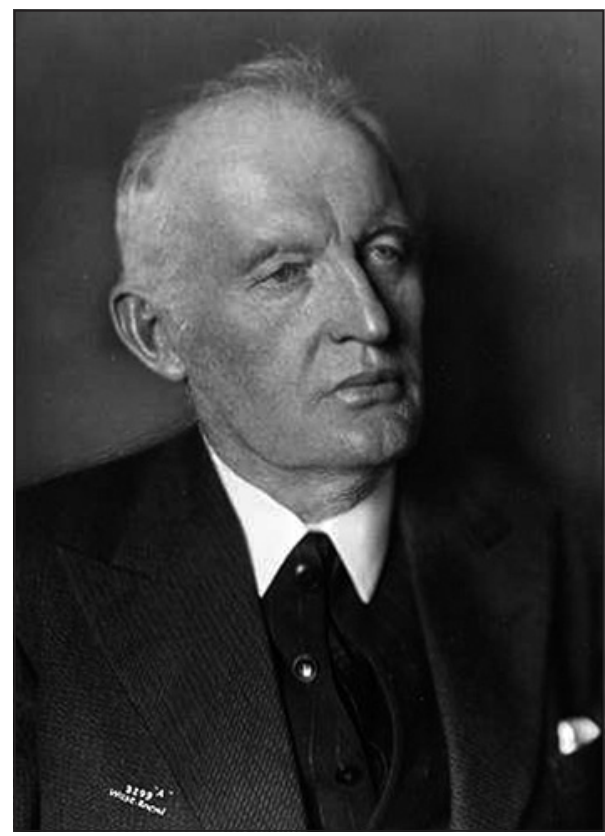

Figura 1. Edvard Munch a los 67 años (1930).

dad bipolar que incluso motivó hospitalizaciones psiquiátricas, (en el caso de Van Gogh se mantiene el debate sobre la verdadera naturaleza de su enfermedad). Coincidieron en su pasión por las mujeres y las relaciones amorosas tormentosas y también en el obsesivo y psicótico afán de autorretratarse. El mismo Munch pintó aproximadamente 50 autorretratos a los largo de su vida. Mucho se especula sobre las razones que tuvo para retratarse tanto, pero es muy probable que obedezca a la necesidad de tener certeza de su propia existencia, de sentirse y validarse viéndose en una tela o en una foto. Munch fue mucho más afortunado que Van Gogh, al gozar en vida de fama y reconocimiento.

Se ha propuesto que Munch sufrió una enfermedad afectiva bipolar ${ }^{8-10}$. Son sus propias descripciones sobre sus alucinaciones las que alimentan esta teoría, la misma productividad que emana de su obra maestra "El Grito" (1893) (Figura 4) y también la evidencia de una inusual actividad creativa durante un viaje a Europa, el que culminó en un confuso incidente con su amor de entonces Tulla Larsen; con quien tuvo una mórbida relación entre los años 1898 y 1902; luego que ella lo manipulara con una amenaza de suicidio con un arma que se disparó en el forcejeo, terminando con la pérdida de una falange de la mano izquierda de Munch.

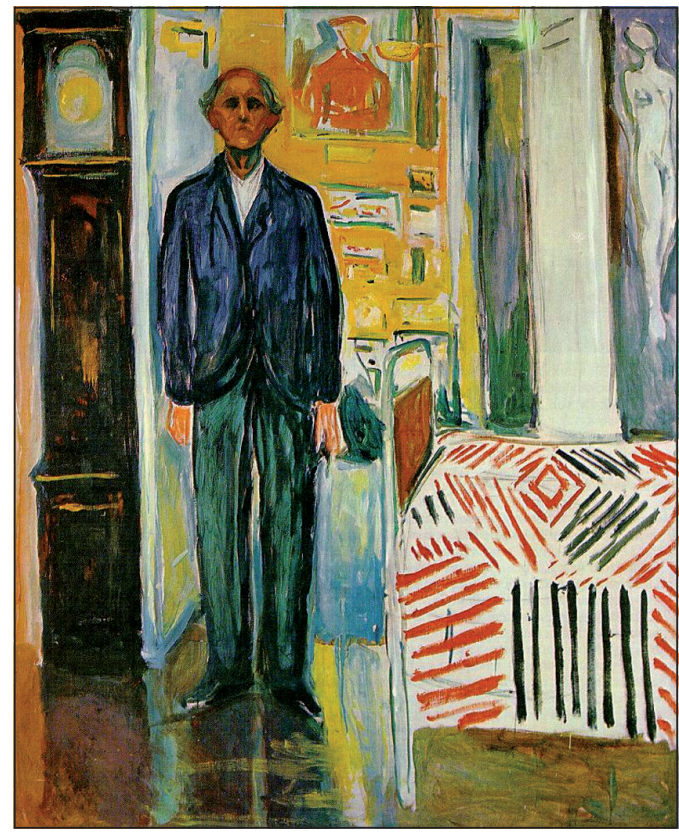

Figura 2. Uno de los últimos autorretratos de Munch.

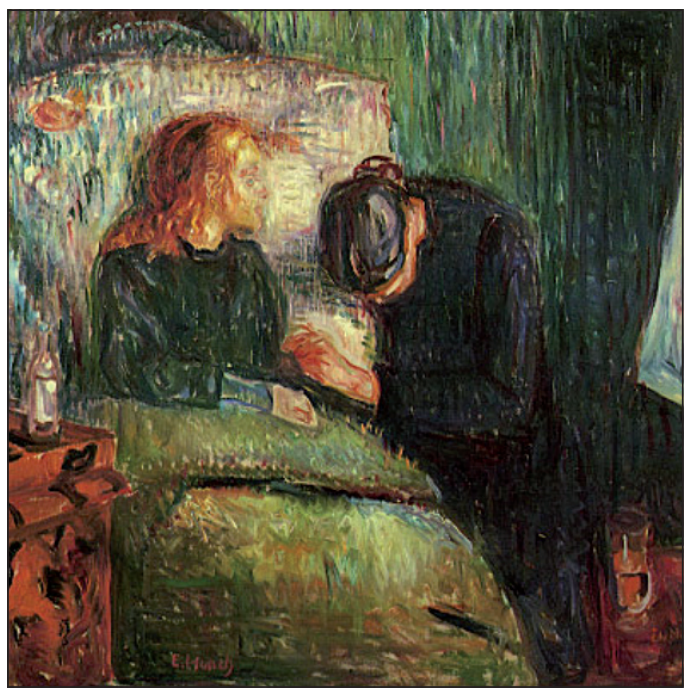

Figura 3. La niña enferma (1885).

El pintor fue hospitalizado en varias oportunidades entre los años 1905 y 1909 por alcoholismo asociado a productividad alucinatoria, ánimo depresivo e ideación suicida.

Estando internado durante varios meses en una clínica danesa aprovecha de autorretratarse en muchísimas fotografías ${ }^{4}$. Estuvo involucrado 


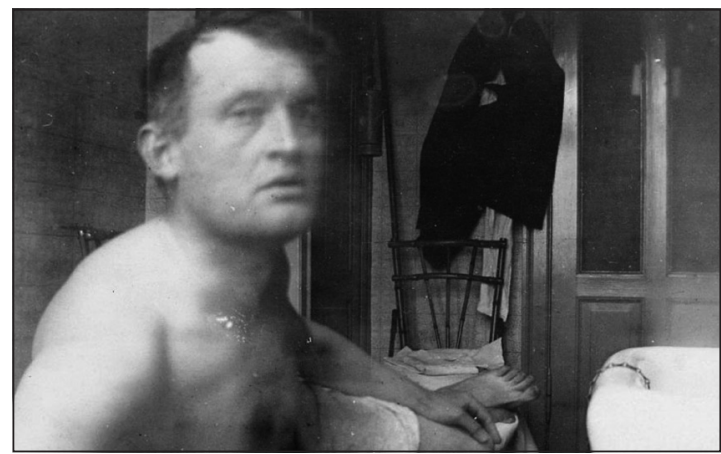

Figura 4. Fotografía tomada por Munch de si mismo, estando hospitalizado en la clínica psiquiátrica del Dr. Jacobsen en 1908; intenta adoptar una postura que semeja el célebre cuadro de J. L. David que representa a Marat asesinado en el baño.

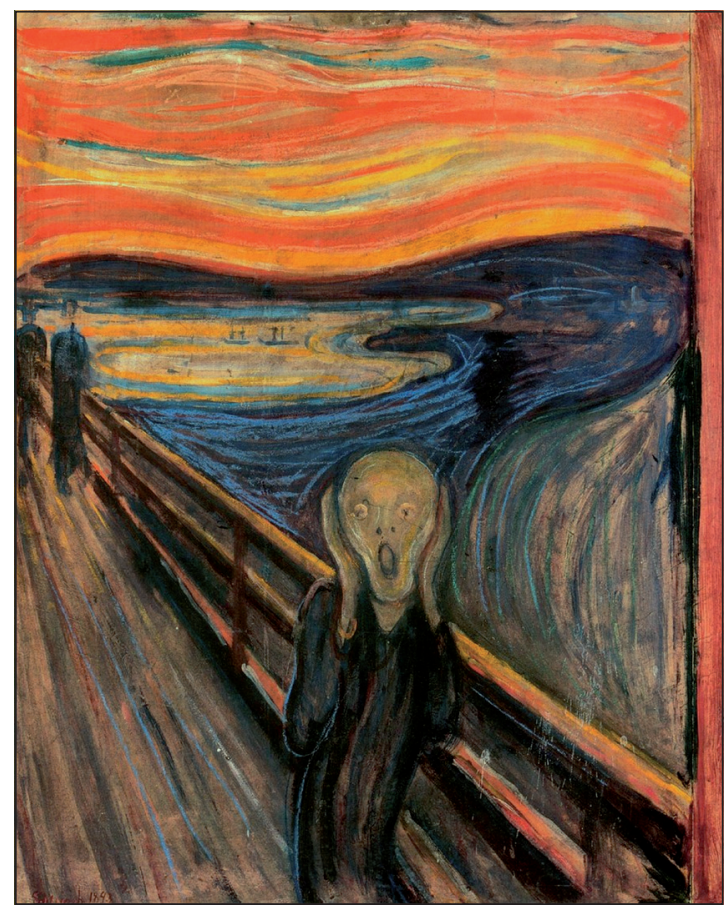

Figura 5. "El Grito" (1893), el cuadro más famoso de Munch.

en hechos de violencia, riñas, peleas y agresiones incluso a otro pintor, debiendo abstenerse de volver a su patria por 4 años. Varios de sus cuadros recuerdan esta disputa.

Es indudable que en Munch hay presencia de perturbaciones permanentes compatibles sólo con una personalidad patológica pero hay elementos que orientan a la existencia de un trastorno del ánimo y que son: la alternancia de períodos de intenso compromiso funcional con otros de actividad relativamente conservada; la marcada inestabilidad afectiva configurada por la sucesión de fases depresivas con períodos de euforia y la aparición en estos períodos de alteraciones de la sensopercepción.

La naturaleza de lo descrito corresponde a lo que hoy se conoce como trastorno afectivo bipolar. La característica principal de esta enfermedad, son las fluctuaciones anímicas con fases maníacas y depresivas que pueden cursar con síntomas psicóticos y que se presentan con manifestaciones conductuales peculiares y con contenidos del pensamiento depresivo o grandioso, según la fase en curso.

Hay argumentos razonables como para postular que Munch poseía una estructura de personalidad de características limítrofes: la existencia de un patrón de inestabilidad afectiva debida a una notable reactividad del estado de ánimo, asociada a descontrol de los impulsos y desajustes conductuales como también a una autoimagen persistentemente inestable, carencia que se expresa en la necesidad de fotografiarse que lo acompañara toda su vida. Sus relaciones interpersonales eran inestables e intensas y era incapaz de mantener una relación de pareja duradera además de presentar frecuentes los episodios de ira y explosividad, rasgos todos muy propios de este tipo de personalidad.

En el año 1981, Munch registró en su diario la concepción de su más famosa obra, "El grito" (Figura 5): "Estaba caminando con 2 amigos. Luego el sol se puso, el cielo bruscamente se tornó color sangre, y sentí algo como el toque de la melancolía. Permanecí quieto, apoyado en una baranda, mortalmente cansado. Sobre el fiordo azul oscuro y de la ciudad, colgaban nubes rojas como sangre. Mis amigos se fueron y yo otra vez me detuve, asustado con una herida abierta en el pecho. Un gran grito atravesó la naturaleza"7,11. El escritor Gerard de Nerval, hizo una descripción similar en una de sus crisis maniacas ${ }^{12}$.

Este cuadro se ha transformado en el símbolo gráfico universal de la angustia existencial que embarga hasta tal punto al protagonista que anula su género pues es casi imposible determinar si se trata de un hombre o de una mujer.

Esta obra que fuera robada desde el Museo de Oslo en el año 2004, fue recuperada muy recientemente y tiene el récord de haber sido vendida en la segunda cifra más alta pagada por una obra de arte. 
Al comienzo del siglo XX y llevado por su permanente curiosidad, compró una cámara fotográfica, la que recién vino a renovar en 1923, con la que registró en forma continua y permanente durante 30 años, numerosos autorretratos, fascinado por dejar constancia de las distintas etapas de su vida y de los cambios que iba experimentando al envejecer. Por el año 1923, se entusiasma también por hacer filmaciones de calles, personas y por supuesto de si mismo ${ }^{6}$.

Así explicaba como concebía su arte: "Yo pinto las lineas y colores que impresionan en mi retina. Pinto de memoria sin agregar nada, sin los detalles que ya no veo enfrente de mí. Esta es la razón de la simplicidad de mis obras, su obvio vacío. Pinto las impresiones de mi infancia, los apagados colores de un día olvidado". "Pintar es lo que el cerebro percibe a través del filtro de los ojos".

En 1930 sufrió una hemorragia del humor vítreo de su ojo derecho, atribuida a hipertensión y estrés, lo que pese a las dificultades que le acarreó, no le impidió seguir pintando ${ }^{1,6}$. En las obras de este período, es posible advertir como aprovechó su enfermedad ocular y las distorsiones visuales que sufría para reflejarlas en sus cuadros. Se dio cuenta que esta afección le permitía experimentar sensaciones visuales imposibles de tener con una visión normal ${ }^{6}$.

Ya en la senectud Munch aún recordaba: "Mi padre era médico militar. Después de la muerte de mi madre, él se convirtió a la religión con una intensidad que aparece haberlo hecho indiferente al éxito mundano y sólo podía asustar a sus hijos." "Mi padre tenía un temperamento difícil, un nerviosismo hereditario, muy angustiado luego de la muerte de mi madre, inició una obsesión religiosa que podría alcanzar las fronteras de la locura mientras caminaba de un lado a otro en su habitación rezando a Dios. Cuando la ansiedad no lo poseía, podría ser como un niño, haciendo bromas y jugar con nosotros... Cuando nos castiga... podría ser casi un loco en su violencia... Enfermedades y la locura fueron los ángeles negros guardianes de mi cuna... siempre sentí que me trataron de manera injusta, sin madre, enfermo y con castigos amenazantes desde el infierno que siguen cerniéndose sobre mi cabeza"7.

\section{Diagnóstico diferencial}

Munch siempre enjuició sus manifestaciones como anormales y es justamente en esta raciona- lización de sus trastornos en que demuestra tener conciencia de enfermedad, actitud que no tiene un paciente esquizofrénico.

Por otra parte, el artista mantuvo una vida creativa hasta el final de sus días, lo que tampoco es compatible con la improductividad de las personas con esquizofrenia.

Como se ha mencionado, el consumo anormal de alcohol era habitual en Munch. La sintomatología que ha quedado registrada también podría ser atribuida a este hábito pero el consumo y la adicción a drogas presentan una relación compleja con los trastornos del espectro bipolar. Es decir, que el uso sustancias no es incompatible con la existencia de un trastorno del ánimo, sino que por el contrario, coexisten y constituyen factores de riesgo entre sí.

\section{Enfermedad y creatividad}

Hemos analizado este controvertido tópico en otra publicación ${ }^{12}$, pero es importante señalar que esta relación se ha propuesto desde la antigüedad. Basta recordar citas de Platón: "La locura que proviene como un regalo del cielo, es el camino por el cual recibimos las más grandes bendiciones. La locura viene desde Dios, mientras que la cordura es meramente humana"12.

Existe la tendencia a asociar los estados y cambios afectivos con genialidad. Así lo refleja el gran artista alemán Alberto Durero en su hermoso grabado llamado "Melancolía" que data de 1514. El ideal del romanticismo en el arte hizo suya esta relación genio-locura-enfermedad. Lord Byron, el poeta inglés, hizo mención al término, "Artista Torturado", diciendo: "Nosotros los artistas somos todos locos, algunos afectados de melancolía, otros de excesiva alegría, pero todos en alguna medida, trastornados"10.

Hay estudios que apoyan y también otros que rechazan la tesis de que la presencia de patología mental influya en tener una capacidad creativa. En un estudio de 1987, la Dra. Nancy Andreason analizó una cohorte de escritores destacados y señaló que tenían significativamente más prevalencia de trastornos afectivos que un grupo control de una inteligencia similar ${ }^{13}$. Andreason encontró que los familiares de primer grado de los escritores tenían tendencia a ser creativos y a presentar un trastorno mental, lo que sugeriría que ambos rasgos estaban 
genéticamente ligados. Una investigación de 2004 hecho por la psicóloga finlandesa Erika Lauronen ${ }^{14}$ mostró que de 13 estudios publicados, excepto uno, todos apoyaban una conexión entre trastorno mental y capacidad creativa. Otros autores, como Nettle, han señalado que esta relación no siempre es causal ya que gran creatividad se puede observar en personas sin patología mental y viceversa ${ }^{15}$. La asociación sería sólo indirecta y reflejaría mecanismos neurobiológicos o genéticos comunes.

Siendo un tópico aún muy controversial, para autores como Barrantes, en esta relación creatividad-psicopatología, es justamente el trastorno afectivo el más asociado a la productividad artística $^{16}$.

Por otro lado, se conoce que la presencia de una actividad anormal del lóbulo frontal y en particular de la corteza prefrontal, es característica tanto de la esquizofrenia como de la depresión manía$\mathrm{ca}^{10}$. La hiperactividad en esta región puede causar que una persona logre conexiones inusuales entre contenidos o ideas aparentemente discordantes, dando lugar a los delirios del esquizofrénico paranoico o del maníaco.

Es planteable que en el proceso creativo se hagan también asociaciones inhabituales lo que lleva a pensar o imaginar de una manera única; sello distintivo de la mente artística ${ }^{10,17}$.

De acuerdo con otro estudio de 2005 realizado por la Dra. Alice Flaherty ${ }^{18}$, el pensamiento creativo, tanto en la depresión maníaca como en la esquizofrenia, también implica una actividad inusual del lóbulo frontal.

Flaherty explica que un déficit de la actividad de la corteza frontal puede disminuir la generación de ideas, en parte a causa de juicios rígidos acerca del real valor de una idea. El destacado neuropsicólogo Kenneth Heilmann también señala que una inusual actividad del lóbulo frontal puede ser responsable de una combinación innovadora de la información almacenada en los lóbulos parietales y temporales lo que favorecería la creatividad ${ }^{17}$.

Los períodos de manía a menudo están asociados al aumento de la inspiración y a mayor productividad. En Munch por ejemplo, vemos en el año 1910 una sorprendente producción artística, posterior a un período depresivo. Por otra parte, algunos artistas maníaco-depresivos logran en sus fases depresivas una mayor productividad porque aumenta su capacidad de introspección, tienen más perspicacia y sensibilidad. Jamison dice "muchos artistas y escritores creen que el caos, el sufrimiento, y los extremos en la experiencia emocional son esenciales no sólo para la condición humana, sino a sus habilidades como artistas. El mejor ejemplo lo da el propio Edvard Munch: "mis problemas son parte mía y de mi arte. Son indistinguibles de mí, un tratamiento destruiría mi arte. Quiero mantener mis sufrimientos".

A los 70 años Munch le dijo a su médico: "la última parte de mi vida ha sido un esfuerzo para estar de pie. Mi camino ha sido siempre a lo largo de un abismo" .

\section{Años finales}

Durante la invasión nazi a Noruega en 1940, los alemanes retiraron sus cuadros de las galerías por considerarlos degenerados y productos de un demente. Munch siempre manifestó su aversión a la ideología nazi. Sin embargo, en la segunda guerra mundial, el pintor se hace mundialmente conocido y en 1942 expuso sus cuadros en Nueva York por primera vez... ${ }^{16}$.

Munch dijo repetidamente: "no quiero morir súbitamente o sin saberlo, quiero tener esa última experiencia también". En sus últimos autorretratos da cuenta de su eventual confrontación con la muerte. Es así como en el último: "Autorretrato entre el reloj y la cama” (1940-3) sitúa su ya frágil figura, entre el símbolo del inexorable paso del tiempo y una cama en la que puede esperar la muerte (Figura 2).

El 23 de enero de 1944 muere en Noruega, solitario, tal como había vivido toda su vida, sin más compañía que la de sus 2 perros, en su casa a las afueras de Oslo.

Al morir, legó más de 1.000 cuadros, 15.400 grabados, 4.500 dibujos y acuarelas y seis esculturas a la ciudad de Oslo, que le rindió homenaje con la inauguración del Museo Munch en 1963.

Munch está entre los artistas más importantes e influyentes del arte moderno. Su obra impactó principalmente en Alemania y en los países Escandinavos donde fue considerado con Van Gogh, como uno de los dos más grandes artistas expresionistas.

La intensidad con la que Munch logra comunicar la angustia del ser humano abrió nuevos caminos al arte. Tal como él decía, sin padecer la enfermedad y sufrimiento, no habría logrado al- 
canzar una obra tan genialmente innovadora. Así entonces, Munch constituye probablemente el mejor ejemplo en la historia del arte de la influencia de un trastorno psíquico en la creación artística, ya que además el propio pintor era plenamente consciente de esta relación.

Agradecimientos: A Osvaldo Gil, Bibliotecario Facultad Medicina Universidad de Chile por el apoyo bibliográfico.

\section{Referencias}

1. Bischoff, Ulrich. Edvard Munch: Cuadros sobre la vida y la muerte. Madrid: Benedikt Taschen Verlag, 2011.

2. Loshack, David. Munch. Madrid: Editorial LIBSA, 1991.

3. Messer, Thomas. Edvard Munch. Madrid: Julio Ollero, 1991.

4. Heller RH: Munch. His Life and Work. London, Calmann and Cooper Ltd., 1984.

5. Chilvers I. Oxford Dictionary of Art and Artists. Oxford University Press 2009.

6. Lampe A. Cheroux C. Edvard Munch: The modern Eye. Tate Publishing. Londres 2012.

7. Wight F. Introduction to Edvard Munch. The Kenyon Review, 1950: 12: 457-69.

8. Rothenberg A. Bipolar Illness, Creativity and Treatment
Psychiatric Quarterly 2001: 72.

9. Jamison K. Touched with fire: Manic-Depressive Illness and the Artistic Temperament. (New York: Simon \& Schuster Free Press Paperbacks, 1993), 51.

10. Sussman A. Me Sussman A. Mental Ilness and Creativity: a neurological view of the "Tortured Artist". Stanford Journal of Neuroscience 2007; 1: 21-4.

11. Crepaldi G. El siglo XIX en Los Siglos del Arte. Electa Barcelona 2005; 284-5.

12. Miranda M. Escritos en Arte y Medicina. Gaete y Cía Eds. Santiago, 2012.

13. Andreason N. Creativity and Mental Illness: Prevalence Rates in Writers and their First-Degree Relatives. The American Journal of Psychiatry 1987; 144: 1288-92.

14. Lauronen E, Veijola J, Isohanni I, Jones PB, Nieminen P, Isohanni M. Links between creativity and mental disorder. Psychiatry 2004; 67: 91-8.

15. Nettle D. Strong Imagination: Madness, Creativity, and Human Nature. (New York: Oxford University Press, 2001.

16. Barrantes-Vidal N. Creativity and madness revisited from current psychological perspectives. Journal of Consciousness Studies 2004; 11: 58-78.

17. Heilman K, Nadeau D, Beversdorf D. "Creative Innovation: Possible mechanisms". Neurocase 2003; 9: 369-79.

18. Flaherty A. "Frontotemporal and dopaminergic control of idea generation and creative drive". The Journal of Comparitive Neurology 2005: 493, 1: 147-53. 